\title{
Dimensionamento de amostra na estimação da população de corós em áreas de campo nativo e de cultivo no Estado do Rio Grande do Sul
}

\author{
Sample size for estimating population density of white grubs in native and cultivated areas in Rio \\ Grande do Sul State, Brazil
}

\begin{abstract}
Alberto Cargnelutti FilhoI Mariana Alejandra ChermanII Jerson Vanderlei Carús Guedes ${ }^{\text {III }}$ Elder Dal Prá ${ }^{\text {IV }}$ Débora Graciani Schallemberger ${ }^{\text {II }}$ Glauber Renato Stürmer ${ }^{\text {II }}$
\end{abstract}

\section{RESUMO}

É importante quantificar a densidade populacional de corós para definir o momento adequado para o controle da praga. Assim, o objetivo deste trabalho foi determinar o tamanho de amostra (número de trincheiras por hectare) para a estimação da média de densidade populacional de corós em áreas de campo nativo e de cultivo em diferentes locais do Estado do Rio Grande do Sul. Foram coletados dados de densidade populacional de corós, independentemente de espécie, em áreas de campo nativo e de cultivo em 17 locais, por meio de contagens realizadas em trincheiras de $20 \mathrm{~cm} \times 50 \mathrm{~cm} \mathrm{com} 30 \mathrm{~cm}$ de profundidade. Em cada uma das 34 áreas amostradas, calcularam-se medidas de tendência central, de variabilidade, de assimetria e de curtose, e testouse a normalidade dos dados. Em seguida, verificou-se a homogeneidade de variâncias entre as áreas em cada local e entre os locais em cada área. Depois, calculou-se o tamanho de amostra em cada área e local. O tamanho de amostra (número de trincheiras por hectare) para a estimação da média de densidade populacional de corós é dependente da área (campo nativo ou de cultivo) e do local. Para as áreas e os locais estudados, 61 trincheiras por hectare são suficientes para estimar a média de densidade populacional de corós, para um erro de estimação igual a 30\% da média estimada, com grau de confiança de $95 \%$.

Palavras-chave: larvas de solo, erro de estimação, tamanho de amostra, amostragem.

\section{ABSTRACT \\ It is important to quantify the population density of white grub to set the appropriate time to control the pest. The}

\begin{abstract}
aim of this study was to determine the sample size (number of trenches per hectare) to estimate the average population density of white grubs in native and cultivated areas in different locations of Rio Grande do Sul State, Brazil. Data were collected from white grubs' population density in native and cultivated areas of 17 locations, regardless of species, through counts in trenches $20 \mathrm{~cm} \times 50 \mathrm{~cm}$ dug to a depth of $30 \mathrm{~cm}$. In each of the 34 areas sampled, the central tendency, variability, asymmetry and kurtosis was calculated and the normality data were tested. Then it was verified the homogeneity of variance among areas in each locality and among locations in each area and it was determined the sample size to estimate the average population density of white grubs in each area and locality. The sample size (number of trenches per hectare) to estimate the average population density of white grubs is dependent on the area (native or cultivated) and locality. It was concluded that 61 trenches per hectare are enough to predict the average population density of white grubs, with an estimation error equal to $30 \%$ of estimated average, with a degree confidence of $95 \%$.
\end{abstract}

Key words: larvae in soil, error of estimation, sample size, sampling.

\section{INTRODUÇÃO}

Os corós são larvas de besouros pertencentes à família Melolonthidae (Coleoptera: Scarabaeoidea) que vivem no solo e se alimentam de raízes de plantas, causando-lhes danos. Entre as

'Departamento de Fitotecnia, Centro de Ciências Rurais (CCR), Universidade Federal de Santa Maria (UFSM), 97105-900, Santa Maria, RS, Brasil. E-mail: cargnelutti@pq.cnpq.br. Autor para correspondência.

IIPrograma de Pós-graduação em Agronomia, UFSM, Santa Maria, RS, Brasil.

"IIDepartamento de Defesa Fitossanitária, CCR, UFSM, Santa Maria, RS, Brasil.

IV Programa de Pós-graduação em Engenharia Agrícola, UFSM, Santa Maria, RS, Brasil. 
diversas espécies, no Estado do Rio Grande do Sul, são encontrados, mais comumente, o coró-daspastagens (Diloboderus abderus Sturm, 1826), o coródo-trigo (Phyllophaga triticophaga Morón \& Salvadori, 1998) e o coró-pequeno (Cyclocephala flavipennis Arrow, 1914). As espécies apresentam as fases de ovo, larva (coró), pupa e adulto (besouro) (SALVADORI, 1999).

Quantificar a densidade populacional de corós, com uma determinada precisão estipulada pelo pesquisador, deve servir de base às decisões sobre práticas de manejo adequadas. A quantificação da densidade populacional de corós pode ser feita por meio de contagem realizada em trincheiras escavadas no solo. Ao dimensionar o tamanho de amostra (número de trincheiras por hectare) para a estimação da média de densidade populacional de corós, é preciso estabelecer um erro de estimação máximo aceitável com um determinado grau de confiança. Porém, como não há um valor que possa ser definido como erro máximo tolerável, deve ser considerada a possibilidade de obter uma estimativa da média de densidade populacional de corós com a máxima confiabilidade possível.

O tamanho e o número de unidades de amostra de solo (trincheiras) para amostragem de larvas de $3^{\circ}$ ínstar de $\boldsymbol{D}$. abderus foi alvo de estudo de SILVA \& COSTA (1998). Os autores concluíram que o melhor tamanho de unidade de amostra foi $25 \mathrm{~cm}$ x $25 \mathrm{~cm}$, com $30 \mathrm{~cm}$ de profundidade e que são necessárias 67 e 419 amostras por hectare para um nível de erro de baixa precisão (25\%) e de alta precisão (10\% da média), respectivamente. Já SALVADORI (1999) sugere utilizar 16 trincheiras por hectare com área de $25 \mathrm{~cm}$ x $50 \mathrm{~cm}$ e $20 \mathrm{~cm}$ de profundidade para $\boldsymbol{D}$. abderus e oito trincheiras por hectare com área de $25 \mathrm{~cm}$ x $100 \mathrm{~cm}$ e $20 \mathrm{~cm}$ de profundidade para P.triticophaga, sem informar a precisão da estimativa da média.

Pesquisas sobre aspectos biológicos, danos e níveis de controle de D. abderus (Sturm) (SILVA, 1995; SILVA, 1997; SILVA, 2000; SILVA \& COSTA, 2002; SILVA \& BOSS, 2002; GARLET et al., 2009) têm sido realizadas. Porém, há carência de informações sobre o tamanho de amostra (número de trincheiras por hectare) necessário para a quantificação da densidade populacional do complexo de espécies de corós, em áreas de campo nativo e de cultivo em diversos locais do Estado do Rio Grande do Sul.

O objetivo deste trabalho foi determinar o tamanho de amostra (número de trincheiras por hectare) para a estimação da média de densidade populacional de corós em áreas de campo nativo e de cultivo em diferentes locais do Estado do Rio Grande do Sul.

\section{MATERIAL E MÉTODOS}

Foram coletados dados de densidade populacional de corós, em corós $\mathrm{m}^{-2}$, em áreas de campo nativo e de cultivo em 17 locais do Estado do Rio Grande do Sul (Tabela 1), por meio de contagens realizadas em trincheiras escavadas no solo. O número de amostras (trincheiras) e o tamanho da área variou entre as 34 áreas amostradas e, como consequência, as distâncias entre as trincheiras foram distintas.

Em cada local, foi selecionada a área de campo nativo adjacente à área de cultivo. Nas 34 áreas amostradas as dimensões da trincheira foram de $20 \mathrm{~cm}$ de largura x $50 \mathrm{~cm}$ de comprimento com $30 \mathrm{~cm}$ de profundidade. As trincheiras foram escavadas aleatoriamente e distribuídas equidistantemente para contemplar a área a ser amostrada. Em cada unidade de amostra (trincheira), com o auxílio de uma pá de corte, fez-se a escavação de uma trincheira no solo e após foi realizado o destorrroamento deste para a verificação da presença e posterior contagem do número de larvas (corós), independentemente da espécie.

Em cada uma das 34 áreas amostradas, a partir dos dados de densidade populacional de corós, foram calculadas as estatísticas: mínimo ( $\mathrm{min})$, máximo (max), média (m), mediana (med), desvio-padrão (s), variância $\left(\mathrm{s}^{2}\right)$ e coeficientes de variação $(\mathrm{CV})$, de assimetria e de curtose. A seguir, foi verificada a normalidade dos dados, por meio do teste de Kolmogorov-Smirnov (CAMPOS, 1983; SIEGEL \& CASTELLAN JÚNIOR, 2006). Aplicou-se o teste de F (unilateral) aos dados de densidade populacional de corós, para verificar a homogeneidade de variâncias entre as áreas de campo nativo e de cultivo (duas variâncias), em cada local (17 testes) e entre os locais (17 variâncias), em cada uma das áreas (dois testes).

Em cada uma das 34 áreas amostradas, foi calculado o índice de Morisita ( $\mathrm{I}_{\delta}$ ) (MORISITA, 1962) e o parâmetro k da distribuição binomial negativa, por meio, respectivamente, das expressões:

$I_{\delta}=\frac{n\left(\sum_{i=1}^{n} x_{i}^{2}-\sum_{i=1}^{n} x_{i}\right.}{\left(\sum_{i=1}^{n} x_{i}\right)^{2}-\sum_{i=1}^{n} x_{i}}$ e $k=\frac{m^{2}}{\left(s^{2}-m\right)}$, nas quais $n$ é o número de trincheiras, $\mathrm{x}_{\mathrm{i}}$ é o número de corós na iésima trincheira, m é a média amostral e s² é a variância amostral.

Para cada área e local, foi calculado o tamanho de amostra (número de trincheiras, ๆ) para as semiamplitudes do intervalo de confiança (erro de estimação) iguais a: 5, 10, 15, 20, 25 e 30\% (D) da estimativa da média ( $\mathrm{m}$ ) de densidade populacional de corós, em corós $\mathrm{m}^{-2}$, com grau de confiança $(1-\alpha)$ de $95 \%$, por meio das expressões: 
Tabela 1 - Altitude, latitude e longitude, cultura atual, data de coleta, tamanho de área e número de trincheiras (n) para a estimação da densidade populacional de corós, em áreas de campo nativo e de cultivo em 17 locais do Estado do Rio Grande do Sul.

\begin{tabular}{|c|c|c|c|c|c|c|c|}
\hline Local & Altitude $(\mathrm{m})$ & Latitude (S) & Longitude (W) & Cultura atual & Data & Área (ha) & $\mathrm{n}$ \\
\hline \multicolumn{8}{|c|}{ 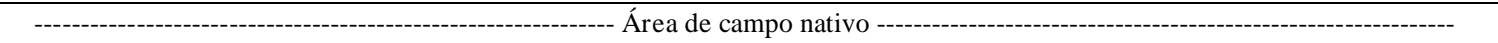 } \\
\hline Caseiros & 770 & $28^{\circ} 15^{\prime}$ & $51^{\circ} 41^{\prime}$ & - & 5/ago/2010 & 5 & 25 \\
\hline Coxilha & 670 & $28^{\circ} 11^{\prime}$ & $52^{\circ} 20^{\prime}$ & - & $10 /$ set $/ 2010$ & 2 & 25 \\
\hline Cruz Alta & 380 & $28^{\circ} 32^{\prime}$ & $53^{\circ} 42^{\prime}$ & - & 27/ago/2009 & 7 & 20 \\
\hline Dois Irmãos das Missões & 535 & $27^{\circ} 39^{\prime}$ & $53^{\circ} 25^{\prime}$ & - & $18 / \mathrm{set} / 2010$ & 3 & 25 \\
\hline Fortaleza dos Valos & 422 & $28^{\circ} 40^{\prime}$ & $53^{\circ} 27^{\prime}$ & - & $9 / \mathrm{set} / 2010$ & 3 & 25 \\
\hline Guabiju & 790 & $28^{\circ} 33^{\prime}$ & $51^{\circ} 39^{\prime}$ & - & 6/ago/2010 & 2 & 25 \\
\hline Ijuí & 400 & $28^{\circ} 31^{\prime}$ & $53^{\circ} 47^{\prime}$ & - & 14/ago/2009 & 2 & 25 \\
\hline Lagoa Vermelha & 680 & $28^{\circ} 29^{\prime}$ & $51^{\circ} 33^{\prime}$ & - & 30/jul/2009 & 9 & 25 \\
\hline Manoel Viana & 140 & $29^{\circ} 13^{\prime}$ & $55^{\circ} 31^{\prime}$ & - & 13/ago/2010 & 4 & 25 \\
\hline Marau & 610 & $28^{\circ} 34^{\prime}$ & $52^{\circ} 07^{\prime}$ & - & 29/jul/2009 & 2 & 20 \\
\hline Nova Palma & 430 & $29^{\circ} 17^{\prime}$ & $53^{\circ} 28^{\prime}$ & - & 25/ago/2010 & 6 & 25 \\
\hline Porto Lucena & 170 & $27^{\circ} 49^{\prime}$ & $54^{\circ} 54^{\prime}$ & - & 21/ago/2010 & 5 & 25 \\
\hline São Francisco de Assis & 180 & $29^{\circ} 18^{\prime}$ & $55^{\circ} 10^{\prime}$ & - & $11 / \mathrm{jul} / 2009$ & 4 & 28 \\
\hline São Luiz Gonzaga & 231 & $28^{\circ} 24^{\prime}$ & $54^{\circ} 56^{\prime}$ & - & $14 / \mathrm{ago} / 2010$ & 5 & 25 \\
\hline Tapejara & 875 & $28^{\circ} 3^{\prime}$ & $52^{\circ} 07^{\prime}$ & - & 28/jul/2009 & 3 & 21 \\
\hline Três Palmeiras & 662 & $27^{\circ} 38^{\prime}$ & $52^{\circ} 49^{\prime}$ & - & $11 /$ set/2010 & 3 & 25 \\
\hline Vacaria & 850 & $28^{\circ} 15^{\prime}$ & $51^{\circ} 16^{\prime}$ & - & 31/jul/2009 & 6 & 25 \\
\hline Caseiros & 770 & $28^{\circ} 15^{\prime}$ & $51^{\circ} 40^{\prime}$ & pousio & 5/ago/2010 & 3 & 25 \\
\hline Coxilha & 670 & $28^{\circ} 11^{\prime}$ & $52^{\circ} 20^{\prime}$ & aveia & $10 / \mathrm{set} / 2010$ & 2 & 25 \\
\hline Cruz Alta & 380 & $28^{\circ} 31^{\prime}$ & $53^{\circ} 41^{\prime}$ & aveia & 27/ago/2009 & 21 & 43 \\
\hline Dois Irmãos das Missões & 535 & $27^{\circ} 38^{\prime}$ & $53^{\circ} 25^{\prime}$ & aveia + azevém & $18 / \mathrm{set} / 2010$ & 8 & 25 \\
\hline Fortaleza dos Valos & 422 & $28^{\circ} 41^{\prime}$ & $53^{\circ} 26^{\prime}$ & aveia & $9 / \mathrm{set} / 2010$ & 5 & 25 \\
\hline Guabiju & 790 & $28^{\circ} 33^{\prime}$ & $51^{\circ} 38^{\prime}$ & azevem & 6/ago/2010 & 9 & 25 \\
\hline Ijuí & 400 & $28^{\circ} 30^{\prime}$ & $53^{\circ} 48^{\prime}$ & pousio & 13/ago/2009 & 13 & 31 \\
\hline Lagoa Vermelha & 680 & $28^{\circ} 38^{\prime}$ & $51^{\circ} 37^{\prime}$ & aveia & 30/jul/2009 & 15 & 30 \\
\hline Manoel Viana & 140 & $29^{\circ} 13^{\prime}$ & $55^{\circ} 31^{\prime}$ & aveia + azevém & 13/ago/2010 & 8 & 25 \\
\hline Marau & 610 & $28^{\circ} 34^{\prime}$ & $52^{\circ} 07^{\prime}$ & azevém & 29/jul/2009 & 5 & 36 \\
\hline Nova Palma & 430 & $29^{\circ} 17^{\prime}$ & $53^{\circ} 28^{\prime}$ & trigo & 25/ago/2010 & 6 & 25 \\
\hline Porto Lucena & 170 & $27^{\circ} 50^{\prime \prime}$ & $54^{\circ} 55^{\prime \prime}$ & trigo & 21/ago/2010 & 6 & 25 \\
\hline São Francisco de Assis & 180 & $29^{\circ} 18^{\prime}$ & $55^{\circ} 17^{\prime}$ & aveia & 11/jul/2009 & 23 & 43 \\
\hline São Luiz Gonzaga & 231 & $28^{\circ} 24^{\prime}$ & $54^{\circ} 56^{\prime}$ & aveia & 14/ago/2010 & 7 & 25 \\
\hline Tapejara & 875 & $29^{\circ} 04^{\prime}$ & $52^{\circ} 07^{\prime}$ & pousio & 28/jul/2009 & 5 & 39 \\
\hline Três Palmeiras & 662 & $27^{\circ} 38^{\prime}$ & $52^{\circ} 50^{\prime}$ & aveia & $11 / \mathrm{set} / 2010$ & 3 & 25 \\
\hline Vacaria & 850 & $28^{\circ} 15^{\prime}$ & $51^{\circ} 17^{\prime}$ & trigo & $31 / \mathrm{jul} / 2009$ & 9 & 35 \\
\hline
\end{tabular}

$\eta=\frac{t_{\alpha / 2}^{2} s^{2}}{(D m)^{2}}($ FONSECA \& MARTINS 1995; BARBETTA

et al., 2004; BUSSAB \& MORETTIN, 2004; SPIEGEL et al., 2004) e $\eta=\frac{\mathrm{t}_{\alpha / 2}^{2}\left(\frac{1}{\mathrm{~m}}+\frac{1}{\mathrm{k}}\right)}{\mathrm{D}^{2}}$ (KARANDINOS, 1976).

Nessas expressões, $\mathrm{t}_{\alpha / 2}$ é o valor crítico da distribuição t de Student, cuja área à direita é igual a $\alpha / 2$, isto é, o valor de $\mathrm{t}$, tal que $\mathrm{P}\left(\mathrm{t}>\mathrm{t}_{\alpha / 2}\right)=\alpha / 2$ com $(\mathrm{n}-1)$ graus de liberdade, com $\alpha=5 \%$ de probabilidade de erro. A fim de definir o tamanho de amostra por hectare (número de trincheiras por hectare), dividiu-se $\eta$ pelo tamanho da área amostrada, em hectares. As análises estatísticas foram realizadas com o auxílio do programa GENES (CRUZ, 2006) e do aplicativo Office Excel.

\section{RESULTADOS E DISCUSSÃO}

A média de densidade populacional de corós $\mathrm{m}^{-2}$, nas áreas de campo nativo, oscilou entre 3,8 e 41,6 e a média dos 17 locais foi 13,1 , enquanto que nas áreas de cultivo variou entre 6,0 e 39,7 com média de 15,5 corós $\mathrm{m}^{-2}$ (Tabela 2). De maneira geral, os resultados sugerem evidências predominantes de maior densidade populacional de corós em área de cultivo em relação à área de campo nativo. No entanto, há locais com evidências contrárias a essa. Portanto, para conclusões 
Tabela 2 - Mínimo (min), máximo (max), média (m), mediana (med), desvio-padrão (s), variância ( $\left.\mathrm{s}^{2}\right)$, coeficiente de variação (CV), assimetria, curtose, resultados do teste de normalidade (Kolmogorov-Smirnov, KS), índice de Morisita $\left(\mathrm{I}_{\delta}\right)$ e parâmetro $\mathrm{k}$ da distribuição binomial negativa, da densidade populacional de corós, em corós $\mathrm{m}^{-2}$, em áreas de campo nativo e de cultivo em 17 locais do Estado do Rio Grande do Sul.

\begin{tabular}{|c|c|c|c|c|c|c|c|c|c|c|c|c|}
\hline Local & $\min$ & $\max$ & $\mathrm{m}$ & med & $\mathrm{s}$ & $\mathrm{s}^{2(1)}$ & $\mathrm{CV}(\%)$ & $\mathrm{A}^{(2)}$ & $\mathrm{C}+3^{(3)}$ & $\mathrm{KS}^{(4)}$ & $\mathrm{I}_{\delta}^{(5)}$ & $\mathrm{k}$ \\
\hline & & & & I & de can & o nativo & & & 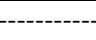 & 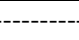 & ------ & --- \\
\hline Caseiros & 0 & 50 & 13,6 & 10 & 16,80 & $282,33^{1 \mathrm{1B}}$ & 123,55 & $1,09 *$ & $2,95^{1 \mathrm{r}}$ & $\mathrm{S}$ & $2,40^{*}$ & 0,69 \\
\hline Coxilha & 0 & 30 & 5,2 & 0 & 7,70 & $59,33^{*}$ & 148,13 & $1,71^{*}$ & $6,30^{*}$ & $\mathrm{~N}$ & $2,94 *$ & 0,50 \\
\hline Cruz Alta & 0 & 20 & 6,5 & 0 & 8,13 & $66,05^{*}$ & 125,04 & $0,77^{\mathrm{s}}$ & $2,00^{\mathrm{B}}$ & $\mathrm{N}$ & $2,35^{*}$ & 0,71 \\
\hline Dois Irmãos das Missões & 0 & 30 & 4,8 & 0 & 8,23 & $67,67^{\mathrm{s}}$ & 171,37 & $1,78^{*}$ & $5,70^{*}$ & $\mathrm{~N}$ & $3,64^{*}$ & 0,37 \\
\hline Fortaleza dos Valos & 0 & 40 & 8,8 & 0 & 12,69 & $161,00^{\mathrm{s}}$ & 144,19 & $1,57^{*}$ & $4,49^{\mathrm{s}}$ & $\mathrm{N}$ & $2,90^{*}$ & 0,51 \\
\hline Guabiju & 0 & 30 & 6,0 & 0 & 9,57 & $91,67^{*}$ & 159,57 & $1,24 *$ & $3,07^{\mathrm{s}}$ & $\mathrm{N}$ & $3,30^{*}$ & 0,42 \\
\hline Ijuí & 0 & 40 & 8,0 & 0 & 10,80 & $116,67^{*}$ & 135,02 & $1,29 *$ & $4,42^{1 \mathrm{~B}}$ & $\mathrm{~N}$ & $2,64^{*}$ & 0,59 \\
\hline Lagoa Vermelha & 0 & 50 & 6,8 & 0 & 12,82 & $164,33^{*}$ & 188,52 & $2,21 *$ & $7,77^{*}$ & $\mathrm{~N}$ & $4,29 *$ & 0,29 \\
\hline Manoel Viana & 10 & 190 & 41,6 & 30 & 37,27 & $1389,00^{*}$ & 89,59 & $2,95^{*}$ & $13,49 *$ & $\mathrm{~N}$ & $1,75^{*}$ & 1,28 \\
\hline Marau & 0 & 40 & 8,5 & 5 & 11,37 & $129,21^{*}$ & 133,73 & $1,52^{*}$ & $5,02^{*}$ & $\mathrm{~S}$ & $2,60^{*}$ & 0,60 \\
\hline Nova Palma & 0 & 120 & 23,2 & 20 & 27,04 & $731,00^{*}$ & 116,54 & $2,08 *$ & $8,90^{*}$ & S & $2,26^{*}$ & 0,76 \\
\hline Porto Lucena & 0 & 30 & 4,0 & 0 & 7,64 & $58,33^{*}$ & 190,94 & $2,20^{*}$ & $7,98^{*}$ & $\mathrm{~N}$ & $4,29 *$ & 0,29 \\
\hline São Francisco de Assis & 0 & 60 & 16,1 & 15 & 15,95 & $254,37^{\mathrm{rS}}$ & 99,24 & $0,82^{1 \mathrm{~K}}$ & $3,38^{15}$ & $\mathrm{~S}$ & $1,89 *$ & 1,08 \\
\hline São Luiz Gonzaga & 0 & 100 & 30,4 & 20 & 32,34 & $1.045,67^{*}$ & 106,37 & $0,79^{\mathrm{5}}$ & $2,31^{1 \mathrm{~B}}$ & $\mathrm{~S}$ & $2,06^{*}$ & 0,91 \\
\hline Tapejara & 0 & 10 & 3,8 & 0 & 4,98 & $24,76^{*}$ & 130,62 & $0,53^{1 \mathrm{~B}}$ & $1,09^{*}$ & $\mathrm{~N}$ & $2,39 *$ & 0,69 \\
\hline Três Palmeiras & 0 & 50 & 15,2 & 10 & 16,61 & $276,00^{*}$ & 109,30 & $0,85^{1 \mathrm{15}}$ & $2,62^{1 \mathrm{I}}$ & S & $2,09 *$ & 0,89 \\
\hline Vacaria & 0 & 110 & 19,6 & 0 & 28,65 & $820,67^{*}$ & 146,16 & $1,66^{*}$ & $5,84^{*}$ & $\mathrm{~N}$ & $3,01 *$ & 0,48 \\
\hline Caseiros & 0 & 60 & 17,2 & 10 & $\begin{array}{r}r a \mathrm{de} \\
19,26\end{array}$ & $\begin{array}{l}\text { ultivo --- } \\
371,00^{\mathrm{s}}\end{array}$ & 111,98 & $0,78^{\mathrm{in}}$ & $2,22^{1 \mathrm{~B}}$ & $\mathrm{~S}$ & $2,15^{*}$ & 0,84 \\
\hline Coxilha & 0 & 40 & 7,6 & 0 & 10,91 & $119,00^{*}$ & 143,54 & $1,57^{*}$ & $5,1^{*}$ & $\mathrm{~N}$ & $2,86^{*}$ & 0,52 \\
\hline Cruz Alta & 0 & 60 & 14,7 & 10 & 16,95 & $287,38^{*}$ & 115,71 & $1,06^{*}$ & $3,10^{\mathrm{B}}$ & $\mathrm{N}$ & $2,24 *$ & 0,79 \\
\hline Dois Irmãos das Missões & 0 & 40 & 10,8 & 10 & 10,77 & $116,00^{1 \mathrm{~s}}$ & 99,73 & $1,13^{*}$ & $4,08^{1 \mathrm{~B}}$ & $\mathrm{~N}$ & $1,87^{*}$ & 1,11 \\
\hline Fortaleza dos Valos & 0 & 50 & 14,8 & 10 & 12,95 & $167,67^{\mathrm{s}}$ & 87,49 & $1,11^{*}$ & $4,05^{15}$ & $\mathrm{~N}$ & $1,67^{*}$ & 1,43 \\
\hline Guabiju & 0 & 130 & 27,2 & 10 & 35,65 & $1.271,00^{*}$ & 131,07 & $1,71^{*}$ & $5,14^{*}$ & $\mathrm{~N}$ & $2,62^{*}$ & 0,59 \\
\hline Ijuí & 0 & 150 & 19,0 & 10 & 30,37 & $922,37^{*}$ & 159,57 & $3,06^{*}$ & $14,34^{*}$ & $\mathrm{~N}$ & $3,42^{*}$ & 0,40 \\
\hline Lagoa Vermelha & 0 & 120 & 39,7 & 30 & 28,46 & $810,23^{*}$ & 71,76 & $0,74^{1 \mathrm{~B}}$ & $3,82^{1 \mathrm{is}}$ & S & $1,47^{*}$ & 2,04 \\
\hline Manoel Viana & 0 & 50 & 8,0 & 10 & 11,18 & $125,00^{*}$ & 139,75 & $2,37^{*}$ & $10,63^{*}$ & S & $2,76^{*}$ & 0,55 \\
\hline Marau & 0 & 20 & 6,9 & 5 & 7,86 & $61,83^{*}$ & 113,23 & $0,61^{1 \mathrm{~B}}$ & $1,91^{1 \mathrm{IS}}$ & $\mathrm{N}$ & $2,11^{*}$ & 0,88 \\
\hline Nova Palma & 0 & 50 & 12,4 & 10 & 10,12 & $102,33^{*}$ & 81,58 & $2,36^{*}$ & $10,71^{*}$ & $\mathrm{~N}$ & $1,56^{*}$ & 1,71 \\
\hline Porto Lucena & 0 & 90 & 32,8 & 30 & 26,38 & $696,00^{*}$ & 80,43 & $0,48^{15}$ & $2,19^{\mathrm{s}}$ & S & $1,59 *$ & 1,62 \\
\hline São Francisco de Assis & 0 & 60 & 13,0 & 10 & 13,72 & $188,26^{1 \mathrm{~B}}$ & 105,36 & $1,28^{*}$ & $4,98^{*}$ & $\mathrm{~N}$ & $2,01^{*}$ & 0,97 \\
\hline São Luiz Gonzaga & 0 & 30 & 7,2 & 10 & 8,43 & $71,00^{*}$ & 117,03 & $1,05^{*}$ & $3,66^{\mathrm{B}}$ & $\mathrm{N}$ & $2,19^{*}$ & 0,81 \\
\hline Tapejara & 0 & 90 & 12,1 & 0 & 19,89 & $395,68^{*}$ & 165,06 & $2,30 *$ & $8,81^{*}$ & $\mathrm{~N}$ & $3,58 *$ & 0,38 \\
\hline Três Palmeiras & 0 & 20 & 6,0 & 0 & 8,66 & $75,00^{*}$ & 144,34 & $0,92 *$ & $1,98^{\mathrm{rg}}$ & $\mathrm{N}$ & $2,85^{*}$ & 0,52 \\
\hline Vacaria & 0 & 60 & 13,7 & 10 & 15,92 & $253,45^{*}$ & 116,08 & $1,25^{*}$ & $4,08^{15}$ & $\mathrm{~S}$ & $2,24 *$ & 0,78 \\
\hline
\end{tabular}

(1) * Variâncias entre as áreas, em cada local, são heterogêneas pelo teste $\mathrm{F}$ unilateral, em nível de $5 \%$ de probabilidade. ns variâncias homogêneas.

(2) * Assimetria difere de zero, pelo teste t, em nível de $5 \%$ de probabilidade. ${ }^{\text {ns }}$ Não-significativo.

(3) * Curtose difere de três, pelo teste t, em nível de 5\% de probabilidade. ${ }^{\mathrm{ns}}$ Não-significativo.

${ }^{(4)} \mathrm{S}=$ Distribuição normal, pelo teste Kolmogorov-Smirnov ( $\left.\mathrm{P}>0,05\right)$. $\mathrm{N}=$ Não normal $(\mathrm{P} \leq 0,05)$.

(5) * Índice de Morisita difere de um, pelo teste $\chi^{2}$, em nível de $5 \%$ de probabilidade.

definitivas sobre possíveis diferenças de densidade populacional de corós em áreas de campo nativo e de cultivo nesses locais, estudos mais detalhados devem ser realizados e não são alvos deste trabalho.

Os escores da mediana revelam que, em 13 áreas, pelo menos em 50\% das trincheiras, não foram encontrados corós. Por outro lado, nas demais 21 áreas, cujo valor da mediana foi superior a zero, foram encontrados corós em pelo menos $50 \%$ das trincheiras (Tabela 2). Ainda, os resultados evidenciam predominância de trincheiras com ausência de corós, nas áreas de campo nativo (10 áreas com mediana igual a zero) em relação às áreas de cultivo (3 áreas com mediana igual a zero). Em 32 áreas amostradas $(94,1 \%)$, 
a mediana foi inferior à média. Esses resultados estão de acordo com a afirmação de que, em distribuições assimétricas positivas, a mediana é inferior à média (FONSECA\& MARTINS 1995; BARBETTAet al., 2004). Portanto, de maneira geral, os menores escores da mediana em relação à média, a distribuição assimétrica positiva dos dados (coeficiente de assimetria $>0$ ) e o grau de achatamento da distribuição, preferencialmente leptocúrtica (curtose >3), são indicativos de afastamento dos dados à distribuição normal.

Entre os 17 locais, nas áreas de campo nativo, o coeficiente de variação oscilou entre $89,59 \%$ em Manoel Viana e 190,94\% em Porto Lucena, enquanto que, nas áreas de cultivo, variou entre 71,76\% em Lagoa Vermelha e 165,06\% em Tapejara (Tabela 2). A ausência de corós em determinadas trincheiras tem contribuição importante nos elevados valores de coeficiente de variação. A média dos coeficientes de variação dos locais foi de 136,35 e 116,69\%, respectivamente, para as áreas de campo nativo e de cultivo, sugerindo que o tamanho de amostra (número de trincheiras por hectare), para obtenção de estimativas com uma mesma precisão, deve ser diretamente proporcional a essa variabilidade. Por outro lado, estimativas obtidas a partir de um tamanho de amostra único apresentariam menor precisão para as áreas de campo nativo em relação às áreas de cultivo.

O teste de Kolmogorov-Smirnov revelou que em 11 áreas amostradas os dados de densidade populacional de corós se ajustaram à distribuição normal ( $\mathrm{P}>0,05)$ (Tabela 2). Por outro lado, em 23 áreas, a não aderência dos dados à distribuição normal $(\mathrm{P} \leq 0,05)$, esteve associada às distribuições, predominantemente, assimétricas positivas $(\mathrm{P} \leq 0,05) \mathrm{e}$ com curtose $>3(\mathrm{P} \leq 0,05)$, além da maior magnitude da média em relação à mediana. $\mathrm{O}$ número de amostras (trincheiras) oscilou entre 20 e 43 e a média entre as 34 áreas amostradas foi de 27,1 trincheiras (Tabela 1). De acordo com o teorema limite central, mesmo que a população básica seja não normal, a distribuição da média amostral será aproximadamente normal para amostras superiores a 30 observações (FONSECA \& MARTINS, 1995; BUSSAB \& MORETTIN, 2004). Então, de maneira geral, diante das considerações em relação à normalidade e ao número de trincheiras, podese inferir que os dados de densidade populacional de corós, dessas 34 áreas amostradas, oferecem credibilidade ao estudo do tamanho de amostra (SIEGEL \& CASTELLAN JÚNIOR, 2006).

$\mathrm{O}$ teste $\mathrm{F}$, aplicado entre as variâncias de densidade populacional de corós das áreas de campo nativo e de cultivo, em cada local, revelou que estas foram heterogêneas $(\mathrm{P} \leq 0,05) \mathrm{em} 13$ locais $(76,47 \%$ dos locais), indicando que o tamanho de amostra para a estimativa da média de densidade populacional de corós é diferenciado entre as áreas (Tabela 2). Nos outros quatro locais (Caseiros, Dois Irmãos das Missões, Fortaleza dos Valos e São Francisco de Assis), as variâncias foram homogêneas, podendo-se inferir que o uso da média do tamanho de amostra entre as áreas é adequado para estimar a média de densidade populacional de corós nesses locais. Entre as variâncias dos 17 locais, em cada área, o teste $F$ revelou variâncias heterogêneas para as áreas de campo nativo ( $F=56,09, P \leq 0,05)$ e de cultivo ( $F=20,56, P \leq 0,05)$, o que revela a necessidade de determinar o tamanho de amostra em cada local. Então, de maneira geral, os resultados indicam a necessidade de utilizar a maior das 34 variâncias estimadas (34 áreas amostradas) para determinar um tamanho de amostra único para essas áreas e locais, a partir de um erro tolerável e grau de confiança fixo. Por outro lado, a variabilidade existente entre as 34 áreas amostradas possibilita a identificação de áreas e locais com maior e menor tamanho de amostra necessário para estimar a média de densidade populacional de corós.

Nas 34 áreas amostradas, a variância foi maior que a média, o índice de Morisita (MORISITA, 1962) foi superior à unidade $(\mathrm{P} \leq 0,05) \mathrm{e}$ o parâmetro $\mathrm{k}$ da distribuição binomial negativa tendeu a zero $(\mathrm{k} \leq 2,04)$ (Tabela 2). Esses resultados indicam distribuição espacial agregada dos corós em todas as áreas amostradas e, portanto, a expressão apresentada por KARANDINOS (1976) para o cálculo do tamanho de amostra, também é adequada.

As duas expressões utilizadas, neste estudo, para o cálculo do tamanho de amostra apresentaram as mesmas estimativas (Tabela 3), o que confirma que ambas as metodologias são adequadas, quando os insetos se distribuem de forma agregada na área. Assim, em ambas as metodologias, o tamanho de amostra, para a estimação da média de densidade populacional de corós em cada área e local, com semiamplitude do intervalo de confiança igual a $5 \%$ da média estimada, em corós $\mathrm{m}^{-2}$ e grau de confiança de $95 \%$, oscilou entre 58 trincheiras por hectare, na área de cultivo em Lagoa Vermelha, e 2.170 trincheiras por hectare na área de campo nativo em Guabiju (Tabela 3). Portanto, em relação à área de cultivo em Lagoa Vermelha, pode-se inferir, com 95\% de confiança, que o intervalo de confiança da média de densidade populacional de corós obtida com 58 trincheiras por hectare é de $\mathrm{m} \pm 0,05 \mathrm{~m}$. Em outro extremo, a precisão de $\mathrm{m} \pm 0,05$ mé obtida com 2.170 trincheiras por hectare, em relação á area de campo nativo em Guabiju. Esses resultados confirmam a variabilidade existente entre áreas dentro dos locais e 
Tabela 3 - Tamanho de amostra (número de trincheiras por hectare, $\eta$ ) para a estimação da média de densidade populacional de corós, em áreas de campo nativo e de cultivo em 17 locais do Estado do Rio Grande do Sul. O $\eta$ foi determinado para as semiamplitudes do intervalo de confiança (erro de estimação) iguais a 5, 10, 15, 20, 25 e 30\% (D) da estimativa da média (m), por meio de duas expressões, nas quais: $\mathrm{t}_{\alpha / 2}$ é o valor crítico da distribuição t de Student $(\alpha=5 \%), \mathrm{s}^{2}$ é a variância amostral e $\mathrm{k}$ é o parâmetro da distribuição binomial negativa.

\begin{tabular}{|c|c|c|c|c|c|c|c|c|c|c|c|c|}
\hline Local & $5 \%$ & $10 \%$ & $15 \%$ & $20 \%$ & $25 \%$ & $30 \%$ & $5 \%$ & $10 \%$ & $15 \%$ & $20 \%$ & $25 \%$ & $30 \%$ \\
\hline & & & $\eta=\frac{\mathrm{t}_{\alpha}^{2}}{(\mathrm{I}}$ & $\frac{s^{2}}{m)^{2}}$ & & --- & & & $\mathrm{t}_{\alpha / 2}^{2}$ & $\left.\frac{1}{n}+\frac{1}{k}\right)$ & & \\
\hline Caseiros & 521 & 131 & 58 & 33 & 21 & 15 & 521 & 131 & 58 & 33 & 21 & 15 \\
\hline Coxilha & 1.870 & 468 & 208 & 117 & 75 & 52 & 1.870 & 468 & 208 & 117 & 75 & 52 \\
\hline Cruz Alta & 392 & 98 & 44 & 25 & 16 & 11 & 392 & 98 & 44 & 25 & 16 & 11 \\
\hline Dois Irmãos das Missões & 1.669 & 418 & 186 & 105 & 67 & 47 & 1.669 & 418 & 186 & 105 & 67 & 47 \\
\hline Fortaleza dos Valos & 1.181 & 296 & 132 & 74 & 48 & 33 & 1.181 & 296 & 132 & 74 & 48 & 33 \\
\hline Guabiju & 2.170 & 543 & 242 & 136 & 87 & 61 & 2.170 & 543 & 242 & 136 & 87 & 61 \\
\hline Ijuí & 1.554 & 389 & 173 & 98 & 63 & 44 & 1.554 & 389 & 173 & 98 & 63 & 44 \\
\hline Lagoa Vermelha & 673 & 169 & 75 & 43 & 27 & 19 & 673 & 169 & 75 & 43 & 27 & 19 \\
\hline Manoel Viana & 342 & 86 & 38 & 22 & 14 & 10 & 342 & 86 & 38 & 22 & 14 & 10 \\
\hline Marau & 1.567 & 392 & 175 & 98 & 63 & 44 & 1.567 & 392 & 175 & 98 & 63 & 44 \\
\hline Nova Palma & 386 & 97 & 43 & 25 & 16 & 11 & 386 & 97 & 43 & 25 & 16 & 11 \\
\hline Porto Lucena & 1.243 & 311 & 139 & 78 & 50 & 35 & 1.243 & 311 & 139 & 78 & 50 & 35 \\
\hline São Francisco de Assis & 415 & 104 & 47 & 26 & 17 & 12 & 415 & 104 & 47 & 26 & 17 & 12 \\
\hline São Luiz Gonzaga & 386 & 97 & 43 & 25 & 16 & 11 & 386 & 97 & 43 & 25 & 16 & 11 \\
\hline Tapejara & 990 & 248 & 110 & 62 & 40 & 28 & 990 & 248 & 110 & 62 & 40 & 28 \\
\hline Três Palmeiras & 679 & 170 & 76 & 43 & 28 & 19 & 679 & 170 & 76 & 43 & 28 & 19 \\
\hline Vacaria & 607 & 152 & 68 & 38 & 25 & 17 & 607 & 152 & 68 & 38 & 25 & 17 \\
\hline Caseiros & 713 & 179 & 80 & 45 & 29 & 20 & 713 & 179 & 80 & 45 & 29 & 20 \\
\hline Coxilha & 1.756 & 439 & 196 & 110 & 71 & 49 & 1.756 & 439 & 196 & 110 & 71 & 49 \\
\hline Cruz Alta & 104 & 26 & 12 & 7 & 5 & 3 & 104 & 26 & 12 & 7 & 5 & 3 \\
\hline Dois Irmãos das Missões & 212 & 53 & 24 & 14 & 9 & 6 & 212 & 53 & 24 & 14 & 9 & 6 \\
\hline Fortaleza dos Valos & 261 & 66 & 29 & 17 & 11 & 8 & 261 & 66 & 29 & 17 & 11 & 8 \\
\hline Guabiju & 326 & 82 & 37 & 21 & 14 & 10 & 326 & 82 & 37 & 21 & 14 & 10 \\
\hline Ijuí & 327 & 82 & 37 & 21 & 14 & 10 & 327 & 82 & 37 & 21 & 14 & 10 \\
\hline Lagoa Vermelha & 58 & 15 & 7 & 4 & 3 & 2 & 58 & 15 & 7 & 4 & 3 & 2 \\
\hline Manoel Viana & 416 & 104 & 47 & 26 & 17 & 12 & 416 & 104 & 47 & 26 & 17 & 12 \\
\hline Marau & 423 & 106 & 47 & 27 & 17 & 12 & 423 & 106 & 47 & 27 & 17 & 12 \\
\hline Nova Palma & 189 & 48 & 21 & 12 & 8 & 6 & 189 & 48 & 21 & 12 & 8 & 6 \\
\hline Porto Lucena & 184 & 46 & 21 & 12 & 8 & 6 & 184 & 46 & 21 & 12 & 8 & 6 \\
\hline São Francisco de Assis & 79 & 20 & 9 & 5 & 4 & 3 & 79 & 20 & 9 & 5 & 4 & 3 \\
\hline São Luiz Gonzaga & 334 & 84 & 38 & 21 & 14 & 10 & 334 & 84 & 38 & 21 & 14 & 10 \\
\hline Tapejara & 894 & 224 & 100 & 56 & 36 & 25 & 894 & 224 & 100 & 56 & 36 & 25 \\
\hline Três Palmeiras & 1.184 & 296 & 132 & 74 & 48 & 33 & 1.184 & 296 & 132 & 74 & 48 & 33 \\
\hline Vacaria & 248 & 62 & 28 & 16 & 10 & 7 & 248 & 62 & 28 & 16 & 10 & 7 \\
\hline
\end{tabular}

entre locais dentro de áreas. Então, 2.170 trincheiras por hectare forneceriam estimativas de média de densidade populacional de corós, com $95 \%$ de confiabilidade de que o erro máximo é $0,05 \mathrm{~m}$, independentemente da área e local.

Do ponto de vista prático, amostrar 2.170 trincheiras por hectare para estimar a média de densidade populacional de corós é difícil. Assim, menores tamanhos de amostra foram determinados com base em semiamplitudes do intervalo de confiança iguais a 10, 15, 20, 25 e $30 \%$ de $m$ (Tabela 3). Não cabe aqui o julgamento do erro de estimação máximo aceitável, ficando essa tarefa ao usuário dessas informações, de acordo com a disponibilidade de dados e a precisão desejada. Portanto, tomando-se como referência o maior tamanho de amostra (área de campo 
nativo em Guabiju), pode-se inferir, com $95 \%$ de confiança, que, com o uso de 61 trincheiras por hectare, o erro máximo na estimativa da média $(\mathrm{m})$ de densidade populacional de corós será de $\pm 30 \%$ de $\mathrm{m}$, independentemente da área e local.

Embora a generalização, a partir desse conjunto de dados, não pode ser realizada, há evidências de maior tamanho de amostra (número de trincheiras por hectare) para a estimação da média de densidade populacional de corós em áreas de campo nativo em relação à área de cultivo. Estudos em mais locais, mais áreas dentro de cada local e ainda com diferentes dimensões de trincheiras, diferentes épocas do ano e em diferentes culturas, são importantes para elucidar inferências generalizadas.

\section{CONCLUSÃO}

O tamanho de amostra (número de trincheiras por hectare) para a estimação da média de densidade populacional de corós no Estado do Rio Grande do Sul é dependente da área (campo nativo ou de cultivo) e do local. Para as áreas e os locais estudados, 61 trincheiras por hectare são suficientes para estimar a média de densidade populacional de corós, para um erro de estimação igual a 30\% da média estimada, com grau de confiança de $95 \%$.

\section{AGRADECIMENTOS}

Ao Conselho Nacional de Desenvolvimento Científico e Tecnológico $(\mathrm{CNPq})$ e à Coordenação de Aperfeiçoamento de Pessoal de Nível Superior (CAPES), pelas bolsas concedidas.

\section{REFERÊNCIAS}

BARBETTA, P.A. et al. Estatística para cursos de engenharia e informática. São Paulo: Atlas, 2004. 410p.

BUSSAB, W.O.; MORETTIN, P.A. Estatística básica. 5.ed. São Paulo: Saraiva, 2004. 526p.

CAMPOS, H. de Estatística experimental não-paramétrica. 4.ed. Piracicaba: Departamento de Matemática e Estatística ESALQ, 1983. 349p.

CRUZ, C.D. Programa genes: estatística experimental e matrizes. Viçosa: UFV, 2006. 285p.

FONSECA, J.S.; MARTINS, G.A. Curso de estatística. 5.ed. São Paulo: Atlas, 1995. 317p.

GARLET, J. et al. Danos provocados por coró-das-pastagens em plantas de eucalipto. Ciência Rural, v.39, p.575576, 2009. Disponível em: <http://www.scielo.br/ scielo.php? script =sci_arttext\&pid=S $0103-$ $84782009000200043 \& 1 n g=p t \& n r m=i s o>$. Acesso em: 31 out. 2010. doi: 10.1590/S0103-84782008005000079.
KARANDINOS, M.G. Optimal sample size and comments on some published formulae. Bulletin of the Entomological Society of America, v.22, p.417-421, 1976.

MORISITA, M. Id-index, a measure of dispersion of individuals. Researches on Population Ecology, v.4, p.1-7, 1962.

SALVADORI, J.R. Manejo de corós em cereais de inverno. Passo Fundo: Embrapa Trigo, Comunicado Técnico Online. n.3, p.1-4, 1999. Disponível em: <http://www.cnpt.embrapa.br/ biblio/p_co03.htm>. Acesso em: 31 out. 2010.

SIEGEL, S.; CASTELLAN JÚNIOR, N.J. Estatística nãoparamétrica para ciências do comportamento. 2.ed. Porto Alegre: Artmed, 2006. 448p.

SILVA, M.T.B.da. Aspectos biológicos, danos e controle de Diloboderus abderus sturm, 1826 (Coleoptera: melolonthidae) em plantio direto. Ciência Rural, v.25, p.343-344, 1995. Disponível em: <http://www.scielo.br/ scielo.php? script=sci_arttext \& pid=S 0103 $84781995000200029 \& 1 \mathrm{ng}=\mathrm{pt} \& \mathrm{nrm}=\mathrm{iso}>$. Acesso em: 31 out. 2010. doi: 10.1590/S0103-84781995000200029.

SILVA, M.T.B.da. Controle de larvas de Diloboderus abderus Sturm (Coleoptera: Melolonthidae) via tratamento de sementes de trigo com inseticidas em plantio direto. Anais da Sociedade Entomológica do Brasil, v.29, p.123-129, 2000. Disponível em: <http://www.scielo.br/scielo.php?script=sci_arttext\&pid=S0301$80592000000100015 \& \operatorname{lng}=$ pt\&nrm=iso $>$. Acesso em: 31 out. 2010. doi: 10.1590/S0301-80592000000100015.

SILVA, M.T.B.da. Níveis de controle de Diloboderus abderus (Sturm) em trigo no plantio direto. Anais da Sociedade Entomológica do Brasil, v.26, p.435-440, 1997. Disponível em: <http:// www.scielo.br/scielo.php? script=sci_arttext\&pid=S0301 $80591997000300004 \& \operatorname{lng}=$ pt\&nrm=iso $>$. Acesso em: 31 out. 2010. doi: 10.1590/S0301-80591997000300004.

SILVA, M.T.B. da.; BOSS, A. Controle de larvas de Diloboderus abderus com inseticidas em trigo. Ciência Rural, v.32, p.191-195, 2002. Disponível em: <http://www.scielo.br/ scielo.php? script $=$ sci_arttext \& pid $=$ S $0103-$ $84782002000200002 \& \operatorname{lng}=$ pt\&nrm=iso $>$. Acesso em: 31 out. 2010. doi: 10.1590/S0103-84782002000200002.

SILVA, M.T.B.da.; COSTA, E.C. Nível de controle de Diloboderus abderus em aveia preta, linho, milho e girassol. Ciência Rural, v.32, p.7-12, 2002. Disponível em: <http:// www.scielo.br/scielo.php? script $=$ sci_arttext $\&$ pid $=$ S0 103 $84782002000100002 \& \operatorname{lng}=$ pt\&nrm=iso $>$. Acesso em: 31 out. 2010. doi: 10.1590/S0103-84782002000100002.

SILVA, M.T.B.da.; COSTA, E.C. Tamanho e número de unidades de amostra de solo para amostragem de larvas de Diloboderus abderus (Sturm) (Coleoptera: Melolonthidae) em plantio direto. Anais da Sociedade Entomológica do Brasil, v.27, p.193-197, 1998. Disponível em: <http://www.scielo.br/ scielo.php? script=sci_arttext\&pid=S 0301 $80591998000200004 \& \operatorname{lng}=$ pt\&nrm=iso $>$. Acesso em: 31 out. 2010. doi: 10.1590/S0301-80591998000200004.

SPIEGEL, R.A. et al. Probabilidade e estatística. 2.ed. Porto Alegre: Bookman, 2004. 398p. 